Alabbas, A. R., Hassnawi, L.A., Ilyas, M., Pervaiz, H., Abbasi, Q.

H. and Bayat, O. (2020) Performance enhancement of safety message communication via designing dynamic power control mechanisms in vehicular ad hoc networks. Computational Intelligence, 37(3), pp. 1286-1308. (doi: 10.1111/coin.12367)

The material cannot be used for any other purpose without further permission of the publisher and is for private use only.

There may be differences between this version and the published version. You are advised to consult the publisher's version if you wish to cite from it.

This is the peer reviewed version of the following article:

Alabbas, A. R., Hassnawi, L.A., Ilyas, M., Pervaiz, H., Abbasi, Q.

H. and Bayat, O. (2020) Performance enhancement of safety message communication via designing dynamic power control mechanisms in vehicular ad hoc networks. Computational Intelligence, 37(3), pp.

1286-1308, which has been published in final form at: 10.1111/coin.12367

This article may be used for non-commercial purposes in accordance with Wiley Terms and Conditions for Self-Archiving.

https://eprints.gla.ac.uk/216825/

Deposited on: 27 May 2020

Enlighten - Research publications by members of the University of Glasgow 


\title{
Performance Enhancement of Safety Message Communication via Designing Dynamic Power Control Mechanisms in Vehicular Ad Hoc Networks
}

\author{
Amjed Razzaq Alabbas ${ }^{\mathrm{a}}$, L. A. Hassnawi ${ }^{\mathrm{b}}$, Muhammad Ilyas ${ }^{\mathrm{c}}$, Haris Pervaiz ${ }^{\mathrm{d}}$, Qammer H Abbasi ${ }^{\mathrm{e}}$ \\ and Oguz Bayat ${ }^{\mathrm{a}}$
}

a Amjed.R. Alabbas is a Ph.D student at the Institute of Graduate Studies, Altinbas University, 34218 Istanbul, Turkey (e-mail: amjed.alabbas@ogr.altinbas.edu.tr).

${ }^{b}$ L. A. Hassnawi is a Doctor of Computer Engineering at the Ministry of Higher Education and Scientific Research and Science and technology, Baghdad, Iraq. (e-mail: laythmail@yahoo.com).

${ }^{\mathrm{c}} \mathrm{M}$. Ilyas is an Assistance professor at School of Engineering and Natural Sciences, at Altinbas University, 34218 Istanbul, Turkey (e-mail: muhammad.ilyas@altinbas.edu.tr).

${ }^{\mathrm{d}} \mathrm{H}$. Pervaiz is an assistance professor at School of Computing \& Communications, Lancaster University, UK (e-mail: h.b.pervaiz@lancaster.ac.uk).

${ }^{\mathrm{e}}$ Q. H. Abbasi is an assistance professor at School of Engineering, University of Glasgow, UK. (e-mail: Qammer.Abbasi@glasgow.ac.uk). ${ }^{a} \mathrm{O}$. Bayat is a Professor in the Department of Electrical and Electronics Engineering of Altınbas University, 34218 Istanbul, Turkey (e-mail: oguz.bayat@altinbas.edu.tr).

Corresponding author: Amjed Razzaq Alabbas

E-mail: amjed.alabbas@ogr.altinbas.edu.tr

Altinbas university.

Turkey/ Istanbul ,34218.

Mobile: (0090)5537427891 


\begin{abstract}
In vehicular ad hoc networks (VANETs), transmission power is a key factor in several performance measures, such as throughput, delay, and energy efficiency. Vehicle mobility in VANETs creates a highly dynamic topology that leads to a nontrivial task of maintaining connectivity due to rapid topology changes. Therefore, using fixed transmission power adversely affects VANET connectivity and leads to network performance degradation. New cross-layer power control algorithms called (BL-TPC 802.11MAC and DTPC 802.11 MAC) are designed, modeled, and evaluated in this paper. The designed algorithms can be deployed on smart cities, highway and urban city roads. The designed algorithms improve VANET performance by adapting transmission power dynamically to improve network connectivity. The power adaptation is based on inspecting some network parameters, such as node density, network load, and MAC queue state, and then deciding on the required power level. Obtained results indicate that the designed power control algorithm outperforms the traditional 802.11p MAC considering the number of received safety messages, network connectivity, network throughput, and the number of dropped safety messages. Consequently, improving network performance means enhancing the safety of vehicle drivers in smart cities, highway, and urban city.
\end{abstract}

Keywords: VANET; Ad Hoc Network; Cross-Layer Model; Safety Application; Collision Avoidance Application; DSRC;802.11p. 


\section{INTRODUCTION}

A vehicular ad hoc network (VANET) is a type of mobile ad hoc network designed to provide communications among adjacent vehicles and between vehicles and fixed road-side equipment [1]. This network type has been established as an intelligent transportation system (ITS) to improve the performance of the transportation systems. The main ITS aims to improve road safety and reduce traffic congestions, fuel consumption, and waiting times in the urban cities, highways as well as smart cities [2],[3]. In addition to intelligent algorithms, integrating sensing devices, embedded computers, digital maps, global positioning system, and wireless communications devices is helpful for the development of several application types for ITS to improve road safety[4]. The updated information supplied by integrating these systems can be helpful for drivers in acquiring real-time data concerning the road conditions, thus allows them to make fast reactions [5]. In wireless networks, the power of transmission has a significant impact on wireless medium sharing because it is responsible for the determination of the signal strength at the receiver, the range of the transmission, and the interference produced for other network receivers. Thus, the transmission power is considered to be one of the main factors in numerous performance measures, such as delay, throughput, energy efficiency, and connectivity [6]. The reduction in the transmission power level can decrease the energy consumption for communications and increase the spatial reuse of wireless media to enhance the efficiency of wireless networks [7]. However, the increase in the transmission power level also raises the transmission range of the nodes. Thus, the average number of required hops in every route in a network is reduced. Consequently, a decrease in the total delay of the transmission is observed in every route [8]. The density of the vehicle frequently and rapidly changes in VANET due to high node mobility, which leads to changes in vehicle distribution and connectivity graph (network topology) [9]. In heavy traffic congestions, the vehicles could be adjacent to each other in a couple of meters, whereas the distance could reach over a hundred meters on sparsely populated roads. Thus, VANETs face the non-trivial issue of connectivity maintenance because of the rapid topology change. For example, when vehicles are distributed densely in the transmission range of one another, numerous nodes must share the medium. Such sharing causes collisions, contentions, and delays, thus reducing the network capacity. However, some vehicles cannot reach their neighbors beyond the transmission range of each other due to the sparse distribution of vehicles and the use of fixed transmission power (fixed transmission range); thus, some vehicles are isolated, and safety messages and other information cannot be received or sent among the isolated vehicles [10]. Therefore, using a fixed transmission power in vehicles produces a fixed transmission range, which adversely affects VANET connectivity and leads to many performance issues and message overheads [11], [12].

This paper mainly aims to propose a solution for connectivity issues in VANETs by designing a new transmission control power algorithm. The proposed algorithm adjusts the transmission range adaptively according to vehicle density. Such an adjustment aims to maintain vehicle connectivity under different network scenarios to improve the performance of this type of network.

In multi-hop communication applications, many factors affect the communication. However, under normal circumstances, one-hop safety messaging scenarios, such as a collision accident safety application, the transmission power of the node is crucial in reaching all one-hop neighbors. Therefore, the one-hop distance depends on the transmission power that the node uses to transmit the safety message. Therefore, the optimal selection of the transmission power for setting its one-hop distance will be crucial in one-hop connectivity in VANETs. The main key contributions of this paper are summarized as follows.

- Improving the connectivity of VANETs by proposing cross-layer, bi-level, and dynamic transmission power control algorithms depending on the information studied at the application layer. The proposed transmission power algorithms produce a variable transmission range, which leads to network 
connectivity maintenance, with high throughput value, and decreases the number of drooped packets Consequently, the VANET network is improved.

- Comparing and analyzing the performance of the proposed algorithms considering the traditional fixed transmission power algorithm 80.211p MAC and with others available cross layer algorithms.

The rest of this paper is organized as follows. Section 2 presents the related work of other authors. Section 3 describes the dedicated short-range communication (DSRC) overview. Section 4 discusses the problem formulation. Section 5 represents the proposed methodology. Section 6 demonstrates the experimental setup. Section 7 explains the performance evaluation. Section 8 presents a comparison for related broadcast algorithms. Section 9 finally concludes the paper.

\section{RELATED WORK}

Many studies considered the VANET problems and started searching and solving its issues because the most popular problem is selecting the proper transmission ranges of the packet. M. I. Ansari et al.[13] proposed an adjustment transmission power called Adaptive-Transmission-Power Ad Hoc On-Demand Distance Vector (ATP-ADOV) routing protocol. The ATP-AODV controls the transmission power adaptively. The proposed routing protocol reduces the overall energy consumption and improves the life duration of the contributing mobile node, thus enhancing the network lifetime. The results show that a good enhancement on the energy consumption and lifespan of the overall network is observed during the application of the ATP-AODV protocol. M. Abdelkader et al. [14] proposed a new cross-layer design called CLD based on IEEE802.11p. The proposed design evaluates the performance at various transmission models (two-Ray, free space, and log-normal). The output result shows that the packet delivery ratio is satisfactory from the perspective of the proposed approach. The minimum delay percentage is also inversely proportional to the number of vehicles. A. Sarfaaraz et al. [15] introduced a power control algorithm called the CLPC. This cross-layer optimization design collects information regarding the receiver signal of the node by using a hello message. Each node can construct the (average minimum and maximum) ranges by using an adaptive transmission power mechanism. The result shows that the proposed approach demonstrates a typical transmission range and efficient communication. K. Kunavut. [16] suggested an innovative approach for adaptive topology controls to allow every one of the nodes to optimize its transmission range and obtain compromising network connectivity levels simultaneously. Results have shown that the suggested approach outperformed the traditional algorithm considering end-to-end delay, collisions, and energy consumptions. D. B. Rawat Et al. [17] presented an innovative approach to adapt transmission power dynamically and the size of the contention window to enhance the efficiency of information dissemination in VANETs. The presented method includes the mechanism of the enhanced distributed channel access of 802.11e and utilizes a joint method for the adaptation of the transmission power at the physical (PHY) layer and the parameters of quality-of-service at the layer of the media access control (MAC). The obtained results from simulations reveal the realization of a sufficient throughput with lower delays compared with that using the default scheme. in [18] H. Chang et al. proposed a new power control algorithm to adjust the transmission power/range for controlling the congestion in communication in VANETs. The authors showed that the new proposed algorithm gave a high sensibility for the surrounding vehicle's status via maintaining the load in the channel below the threshold. the results showed that the new power control algorithm handle with the congestion in the communication channel fairly to target the allowed threshold in various traffic situations. W. Xue-wen et al. [19] developed an innovative broadcast approach for the VANETs, which is referred to as the transmission range adaptive broadcast (TRAB). They also calculated the waiting time for selecting the nodes of the relay to ensure that fewer relay nodes are utilized to forward emergency packets. Moreover, the 
TRAB ensures the reliability of the dissemination through the adoption of the following two answering mechanism types: the explicit ACK and the implicit ACK. The simulation results indicate that (TRAB) outperformed the available typical algorithms of broadcasts for VANETs according to the broadcast redundancy suppression, reliability, and real-time performance. P. J. Wan et al. [20] demonstrated a range assignment to nodes in VANETs and performed an analysis of the exact asymptotic distributions of a critical radius of transmission for k-connectivity; the following two methods have been implemented: the 1-nearest neighbor range assignments and the uniform assignment range. S. A. A. Shah et.al. [21] designed a multimetric power controller (MPC). this approach depends on uses the channel status and application requirement to estimate the needed transmission power/range to send the safety messages. the results showed that the proposed algorithm gave the best coverage for different messages and in turn, will improve the awareness by minimizing the collision in the beacons. Y. Zhuang et al. [22] presented a time and locationcritical (TLC) model for disseminating EMs. This model facilitates the broadcast of messages of different importance levels to various distances simultaneously. Owing to these characteristics, (TLC) has been wellfitted for the requirements of advanced travel planning and instant collision avoidance in VANETs.

\section{DEDICATED SHORT RANGE COMMUNICATION (DSRC) OVERVIEW}

DSRC [23] can be defined as a wireless radio technology based on Wi-Fi to assist data exchange in a short and high dynamic network. In 1999, the Federal Communication Commission of the United States of America specified $75 \mathrm{MHz}$ of the DSRC spectrum at $5.9 \mathrm{GHz}$, which is used for the communications between the vehicles (V2V) and between the vehicles and the roadside units (V2I) [24]. DSRC was described in [25] as a key technology for the next generation of safety vehicular communication. Broadcasting was considered to be one of the substantial services in DSRC. The technology was designed to be incorporated in the automotive industry. DSRC is a group of protocols and criteria that contains all parts of the layers; its PHY and MAC layers have been defined in IEEE 802.11p, which belongs to the 802.11 family. The DSRC properties are represented in low connection delay and high data transfers [26], [27]. The DSRC contains seven channels (CH172- $\mathrm{CH} 184)$ as shown in Figure 1.

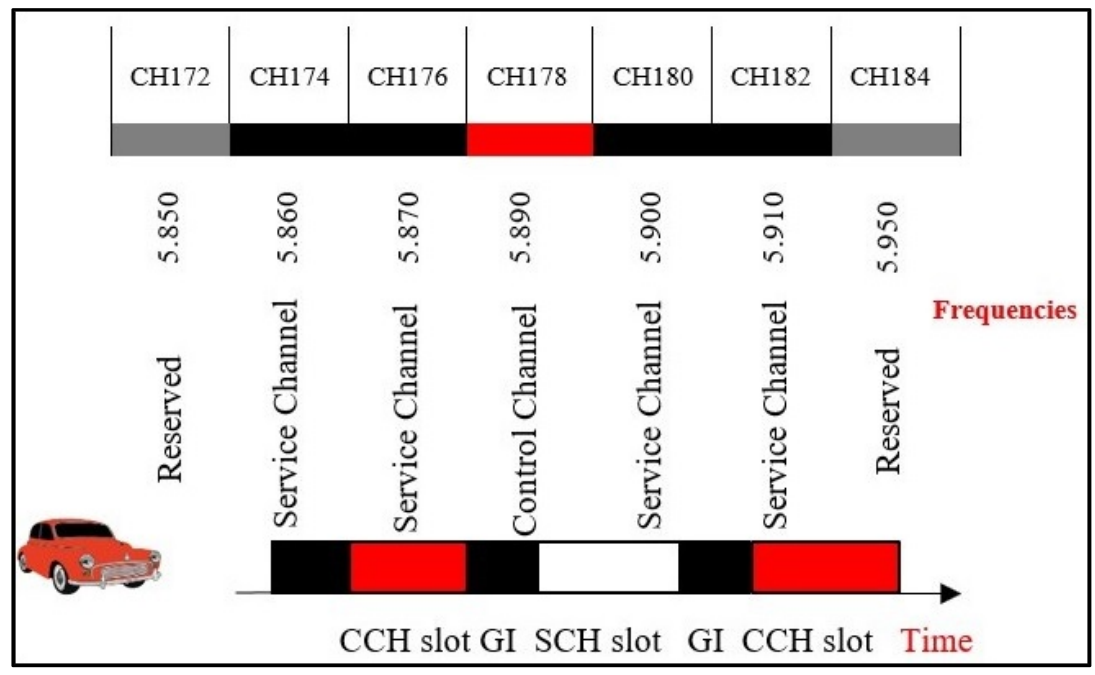

Figure 1 DSRC spectrum frequencies and IEEE1609.4 protocol operations

Each channel reserved $10 \mathrm{MHz}$, while the remaining $5 \mathrm{MHz}$ is reserved for guard band. CCH178 is used as a control channel. The DSRC channels can be summarized into the following two categories: a control channel $(\mathrm{CCH})$ and service channels (SCHs). The 1st and 7th channels are dedicated to applications of high availability low latency and intersection, respectively. The middle (i.e., the 4th channel) is for controlling various values of transmission power and a node, which is not permitted to occupy the channel for more than 
$200 \mu \mathrm{s}$ at each time. The 2nd and 3rd channels are for medium-range services (with a limited transmission power of $33 \mathrm{dBm}$ ) and may be bonded to one $20 \mathrm{MHz}$ channel. The 5th and 6th channels (may also be bonded) are for short-range services (with a limited transmission power equal to $23 \mathrm{dBm}$ ). The main aim is to enable the applications of public safety, which can save lives and enhance traffic flow [28], [29]. The DSRC works with different kinds of data rate transfer, such as 3, 4, 5, 6, 9, 12, 18, 24, and $27 \mathrm{Mbps}$, for the $10 \mathrm{MHz}$ channel, and $6 \mathrm{Mbps}$ is the most optimal data rate transfer [30]. The vehicular network works on DSRC bands, which can be regarded as a key enabler technology for the new marketing of the ITS. The IEEE1609 Standard for Wireless Access in Vehicular Environments (WAVE) [31], which allows the cohabitation of safety/non-safety applications in a vehicular network, was proposed to improve the performance for the multichannel operation of the vehicular network. The limited support of WAVE 1609.4/802.11p under the NS2 (v-2.35) software presents a challenge in evaluating the performance of the 1609.4-based VANETs. The basis of the IEEE 1609 standards family is the IEEE 802.11p standard, which defined PHY and MAC layers and heavily relies on previous standards for wireless LANs [32], [33].

\section{PROBLEM FORMULATION}

The Nakagami propagation model has been comprehensively defined in [34] as a general mathematical radio channel modeling with fading. In comparison with available models (two-ray ground and shadowing), the RF model of Nakagami includes additional configurable parameters to allow close representations of wireless communication channels. This model is capable of modeling from an optimal free space channel to an adequate fading channel on the highway, even to an intensely fading channel in the urban communities. The distribution of Nakagami can be characterized by the following probability density function:

$$
f(x)=\frac{2 m^{m} x^{2 m-1}}{\Gamma(m) \Omega^{m}} \exp \left[\frac{-m x^{2}}{\Omega}\right], x \geq 0, \Omega>0, m \geq 1 / 2,
$$

where $\Omega$ represents the projected distribution value and can be defined as the mean value of the received power, and $m$ represents the parameter of fading or shape and gamma function, which is defined as $\Gamma(m)$. The corresponding power pdf (square of the signal amplitude) at the specified distance may be obtained through a variety of variables and can be specified through the gamma distribution as follows:

$$
p(x)=\left(\frac{m}{\Omega}\right)^{m} \frac{x^{m-1}}{\Gamma(m)} \exp \left[-\frac{m x}{\Omega}\right],-x \geq 0
$$

The IEEE802.11p simulation framework of NS2 supports the RF model of Nakagami. The values of $\mathrm{m}$ and $\Omega$ are the distance functions. Therefore, the Nakagami model can be defined by the following two functions: $\mathrm{m}(\mathrm{d})$ and $\Omega(\mathrm{d})$.

In the free-space model, the Friis transmission equation (3) was utilized to calculate the power received from one of the antennas (with gain G1) in the case of the transmission from another antenna (with gain G2), which is separated by a distance $\mathrm{R}$ and operating at frequency or lambda wavelength. According to the freespace model, received power is generally computed as follows:

$$
P_{r}=\frac{P_{t} \times G_{t} \times G_{r} \times \lambda^{2}}{(4 \times \pi \times d)^{2} \times L}
$$

where $P_{r}$ is the received power, $\mathrm{G}_{t}$ is the transmitter antenna gain, $\mathrm{Gr}$ is the received antenna Gain, $\lambda$ is the wavelength, $\mathrm{L}$ is the system loss, and $\mathrm{d}$ is the distance between the transmitter and receiver. After modifying equation (3) the transmission power can calculate as follows:

$$
P_{t}=\frac{P_{r} \times \mathrm{G}_{t} \times \mathrm{G}_{r} \times \lambda^{2}}{(4 \times \pi \times \mathrm{d})^{2} \times \mathrm{L}},
$$


Under the NS2(V-2.35) implementation of 802.11p, the following expression can be used to resolve the transmission power considering the distance as follows:

$$
\mathrm{P}_{\mathrm{t}}=(3.162 \mathrm{e}-12) / \mathrm{Y} \text {. }
$$

Where 3.162e-12 is the received Signal Strength equivalent to $-85 \mathrm{dBm}$, it is wireless interface sensitivity defined in the standard and $\mathrm{Y}=\left(\mathrm{G}_{\mathrm{t}} * \mathrm{G}_{\mathrm{r}} * \mathrm{M} * \mathrm{M}\right) / 1$. M can be defined as $\lambda /(4 * \pi * \mathrm{~d})$ where $\pi=3.14, \mathrm{G}_{\mathrm{t}}=$ $\mathrm{G}_{\mathrm{r}}=5.118, \mathrm{f}=5.9 \mathrm{e} 9 \mathrm{~Hz}, \mathrm{c}=3.0 \mathrm{e} 8 \mathrm{~m} / \mathrm{s}$ and $\lambda=\mathrm{c} / \mathrm{f}=3.0 \mathrm{e} 8 / 5.9 \mathrm{e} 9=3 / 59$.

\section{PROPOSED METHODOLOGY}

Under a typical VANET scenario, safety applications, such as a "vehicle collision avoidance," can be regarded as a basic application that always runs on vehicles for their safety. A typical "collision avoidance" application is a one-hop-based safety messaging application. Furthermore, most of the safety applications of VANETs are one- or multi-hop broadcast-based applications. Thus, at least one one-hop safety messaging application, such as a "collision avoidance" application, is assumed to run on the network. The optimal selection of the transmission power for setting its one-hop distance will be crucial in one-hop connectivity in VANETs. If a node transmits an emergency message, then at least one of its neighbors should receive that message. The sent node may obtain help from the received node or the received node may broadcast that message to their neighbors. Therefore, the transmission power used for one-hop message transmission is crucial for the efficient collision-free operation of VANETs.

Two novel cross-layer design transmission power control algorithms are proposed in this paper based on transmission power/range to maintain connectivity between vehicles on roads. These algorithms are as follows:

- Bi-Level Transmission Power Control (BL-TPC)

- Dynamic Transmission Power Control (DTPC)

The cross-layer design for both algorithms is based on the inspection of some network parameters at the application layer and the instruction of the MAC/Physical layers to change the transmission power dynamically considering the values of inspected parameters. The proposed algorithms implemented a neighborhood information gathering mechanism at the application layer. At each node, the "active" number of "current" neighbors of a moving vehicle is collected and the nearest and farthest node/vehicle are resolved only by monitoring the collision avoidance messages from its one-hop neighbors. By contrast, the necessary Tx power needed to transmit the safety message up to a particular distance considering the propagation model in use can be resolved. The transmission power/range of the node at the MAC/Physical Layer can be set on the basis of the instructions received from the application layer. The safety messaging application running on the vehicles will periodically send collision avoidance safety messages. Each safety message broadcasted from the vehicle contains the current location of the vehicle that is transmitting the safety messages.

\section{A. Cross-Layer, Bi-Level Transmission Power Control Algorithm (BL-TPC) 802.11p MAC Algorithm}

The (BL-TPC) algorithm can be applied to each vehicle to increase road safety and save the lives of drivers. BL-TPC can only increase the transmission power by a percentage value. The operational concepts of this algorithm are based on the vehicle movement on the sparse region of the highway due to high speeds. Therefore, the transmission power must be increased. This algorithm is suitable for long vehicles (e.g. trucks and Trailer) which are restricted to move at highways and not allowed to move inside cities, in other words, we can say it is suitable to deploy when increasing $T x$ is much required. In this proposed algorithm, the 
neighborhood density is used as the main parameter to decide the transmission power at each node (vehicle). In the designed algorithm, in addition to the normal function, the safety application running at the vehicle will attempt to estimate the number of neighbors and its distances and predict its movement in a dense or sparse region. If a vehicle realizes that it is moving at a sparse region, then the vehicle will increase its transmission power by instructing the MAC layer to set an appropriately high transmission power. The increased transmission power will facilitate the sending of future messages to a slightly far distance from the sparse region to reach its distant neighbors.

In the designed algorithm, the vehicle starts transmission of the safety message with the default transmission power/range $(250 \mathrm{~m})$. Each broadcasted safety message contains the current location of the vehicle, vehicle direction, vehicle speed, vehicle ID, and timestamp of sending the message as described in (steps 1,2, and 3), as shown in table 1 . The vehicle that sent the safety message starts estimating the number of neighbors and its distances and attempt to predict movement in a dense region or not. If the vehicle detects traffic on the road, then the vehicle will transmit the message at the default transmission power (step 4). The vehicle can realize the environments of the region (dense or sparse) in several ways. If the number of received safety messages is comparatively low or the frequency of the received safety messages is less, then the vehicle can understand its movement in a sparse location. Even after the vehicle realized its movement in a sparse region, parameters must be checked before increasing the transmission power/range as presented in (steps 5 and 7) in Table 1. The node maintains a "recent neighbors list" based on the received messages. Then, a lower number count in the "recent neighbors list" will signify the vehicle movement in a sparse location. The vehicle will inform the MAC/PHY layer to increase its transmission power (default transmission range + $50 \%$ default transmission range) to send its messages to a slightly far distance from the sparse region to reach its distant neighbors (Steps 9 and 10). Tables 1 and 3 show the operation concepts of the BL-TPC algorithm at the sender and receiver side, respectively and figure 2. Shows diagram briefly explains the process at the transmitting end for BL-TPC 802.11p MAC algorithm operation concept.

Table 1 ALGORITHM FOR BL-TPC (SENDER SIDE

Step 1: Create new safety Message

Step 2: Set the time stamp T and message ID to the created message and other message specification

Step 3: Broadcast the message with default transmission power (default Tx Power)

Step 4: Check Neighbor Count and if higher than sparse neighbor GOTO step 3

Step 5: Else check if the time expired without finding any receiver

Step 6: If yes current dropped message and GOTO step 1

Step 7: Else Check if the Tx power larger than Max allowed Tx Power

Step 8: If yes GOTO step 3

Step 9: Else increase Tx power to be (New Tx Range $=$ Default Tx

Range + Default Tx Range / 2).

Step 10:Broadcast the message

Step 11:GOTO Step 1 


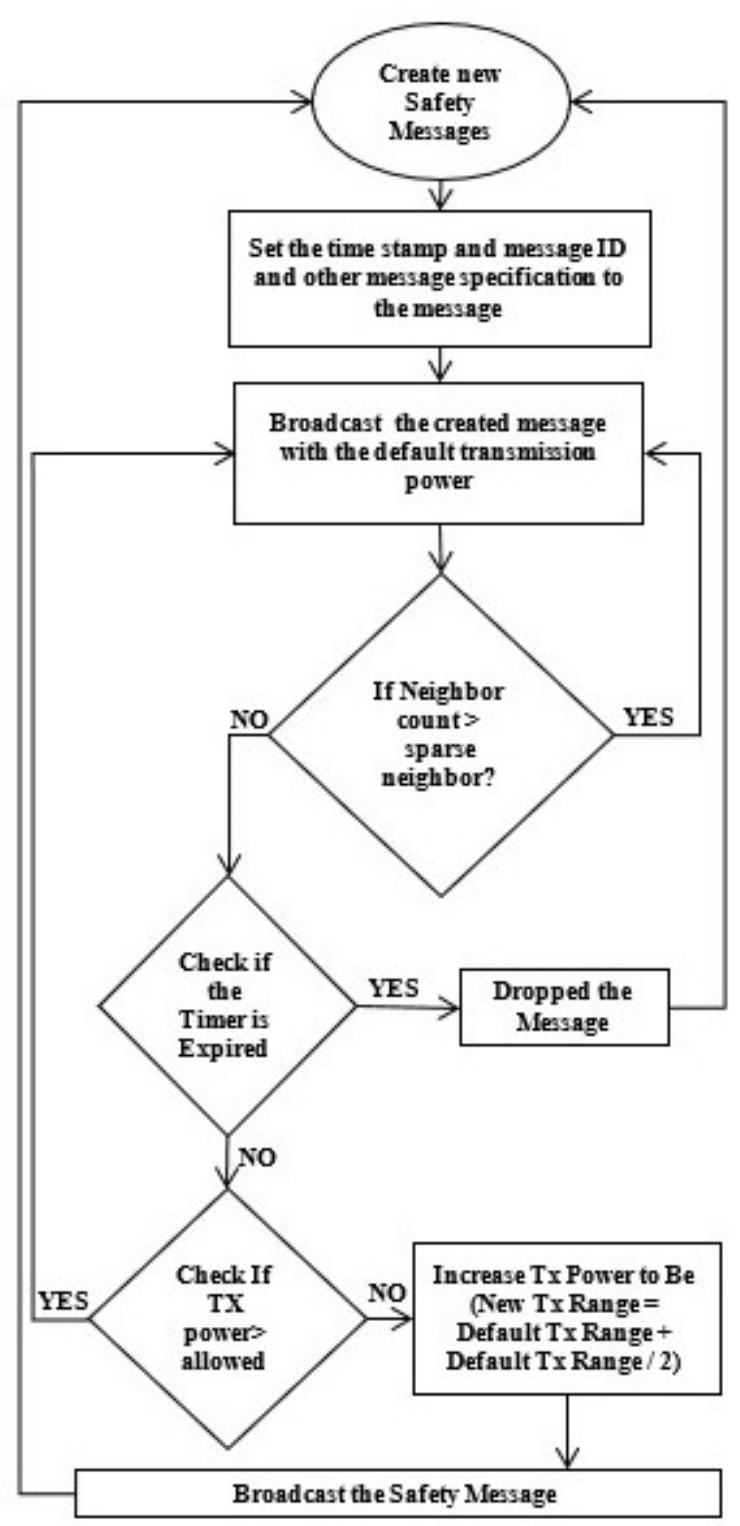

Figure 2. Flowchart for BL-TPC 802.11p MAC Algorithm Operation Concept infrastructure

\section{B. Dynamic Transmission Power Control (DTCP) Algorithm (DTCP) 802.11p MAC Algorithm}

The Dynamic Transmission Power Control (DTPC) algorithm facilitates the flexible transmission of the safety message by the sender node, which may increase or decrease the transmission power/range as needed. This algorithm increases the Tx range to a maximum amount if it senses movement in the sparse region and decreases the Tx range below the default Tx range if it senses a high number of neighbors. Therefore, this power control algorithm is suitable in smart cities/urban cities and in the highways. The power level is increased or decreased dynamically according to the neighborhood density and the operational concept of the DTPC. This algorithm is most suitable for vehicles that move inside cities which also can use the highway for traveling between cities. this algorithm can be used with vehicles restricted to moves in highways. but the minimize option will be useless or rarely, as we mentioned before the sparse area in the highway is dominant, and increasing $\mathrm{Tx}$ is required as in (BL-TPC).

In DTPC, the vehicle starts transmission of the safety message with the default transmission power/range $(250 \mathrm{~m})$. Each broadcasted safety message contains the current location of the vehicle, vehicle direction, vehicle speed, vehicle ID, and timestamp of sending the message as shown in Table 2 (steps 1, 2, and 3). The 
neighborhood density and the interface queue size of the vehicle are used to decide on setting the transmit power level. If the vehicle senses that the queue size is larger than the threshold, then the node is wellconnected and can broadcast the safety message (step 4).

The vehicle that sent the safety message starts estimating the number of neighbors and its distances and attempts to predict the movement in a dense or sparse region. If the creator of the safety message estimates its movement in the dense region by checking the neighbor count (step 5), then the transmitting power will be decreased as needed (step 13); if it estimates movement in a sparse region, then the transmitting power will be increased as needed (step 10). Checking the expiry time is also important because the message will be dropped, and the transmitter will rebroadcast the message until an active receiver is found if the safety message does not find a receiver (steps 6 and 7). The process of changing the transmission power/range of the node at the MAC/PHY layer is based on the instructions received from the application layer. Tables 2 and table 3 show the operation concepts of the DTPC algorithm at the sender and receiver side, respectively and figure 3 shows a diagram briefly explains the process at the transmitting end of DTPC 802.11p MAC Algorithm Operation Concepts. The safety application running on the vehicle will generally receive such safety messages and understand the distance and its nearest vehicle by periodically inspecting the safety messages to resolve the locations of the vehicle that is sending the message.

Table 2 ALGORITHM FOR DTPC (SENDER SIDE)

Step 1: Create new safety Message

Step 2: Set the time stamp T and message ID to the created message and other message specification

Step 3: Broadcast the message with default transmission power (default Tx Power)

Step 4: Check if the Queue Size is larger than the Threshold if yes broadcast the message

Step 5: Else, Check Neighbor Count and if it higher than sparse neighbor Go to step 13

Step 6: Else check if the Time expired without finding any receiver

Step 7: If yes DROP the current message and GOTO step 1

Step 8: Else Check if the Tx power LARGER than Max allowed Tx Power

Step 9: If yes GOTO step 3

Step 10: Else INCREASE Tx power to be (New Tx Range = Default

Tx Range + Default Tx Range / 100* Increment Percentage).

Step 11: Broadcast the message

Step 12: GOTO Step 1

Step 13: Decrease the Tx power level by Default Tx Range - (Default

Tx Range /100* Increment Percentage)

Step 14: Broadcast the message

Step 15: GOTO Step 1 


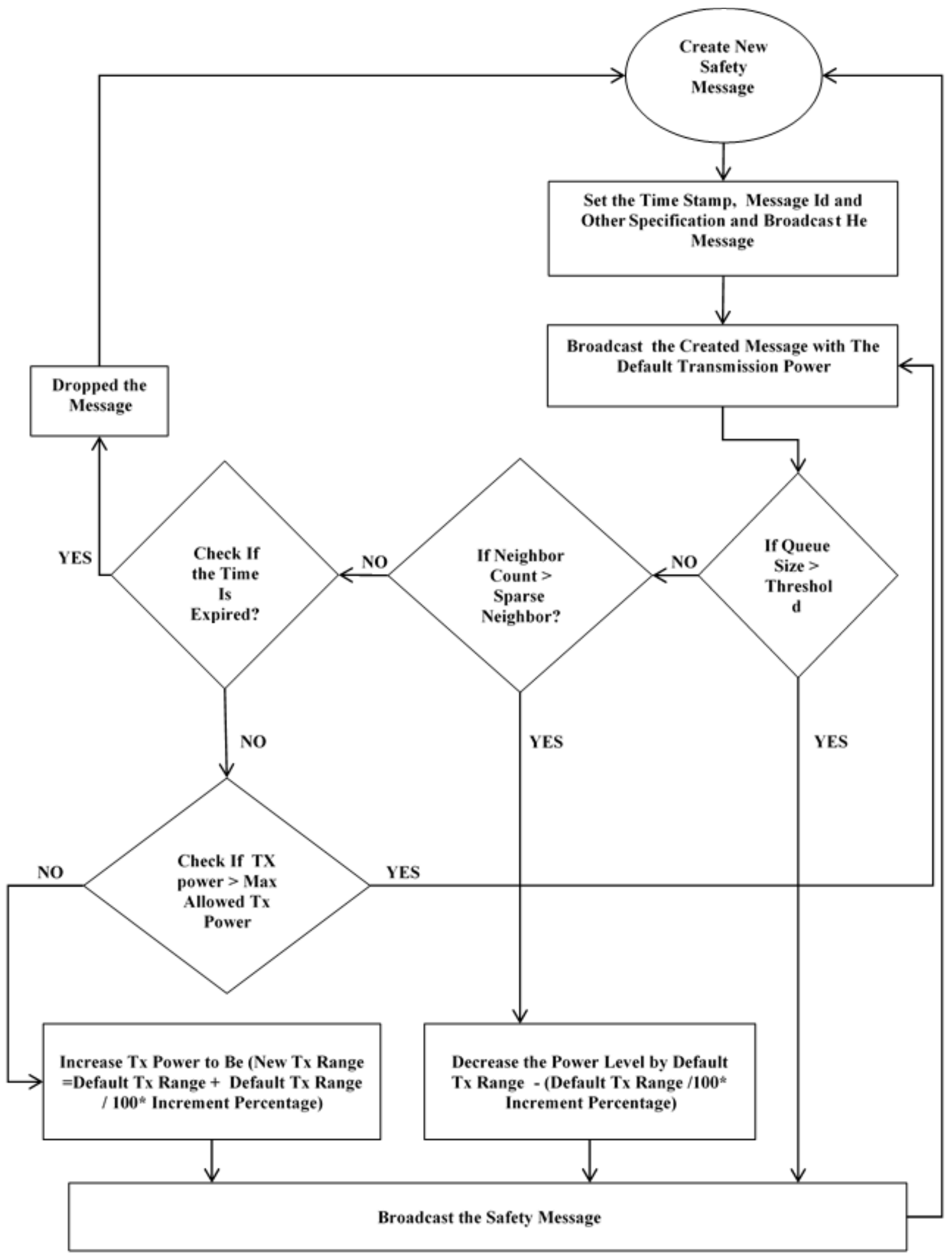

Figure 3. Flowchart for DTPC 802.11p MAC Algorithm Operation Concepts

On receiving each safety message at the application layer, the neighborhood parameters will be updated considering the new safety message content as shown in table 1 (Step 1). The collision avoidance kind of one-hop safety messaging is a basic and unavoidable application that exists on every basic VANET infrastructure (step 2). Table 3 indicates the storage of neighborhood information in a buffer as explained in 
steps 3, 4, and 5. Figure 4 shows diagram briefly explains the process at the receiving end. which explains how the neighborhood information is stored in a buffer. The buffer works as a circular buffer and starts overwriting its old content with a new one once it becomes full. Therefore, the buffer contains the latest neighborhood information collected from the received safety messages.

Table 3 ALGORITHM FOR BL-TPC\& DTPC (RECEIVER SIDE)

Step 1: Vehicle received a packet

Step 2: Do default action (like collision avoidance)

Step 3: Update Neighbor Info Buffer at "Index I"

Step 4: If the buffer full set the buffer flag as true and free the message

Step 5: Else reset the buffer index and free message

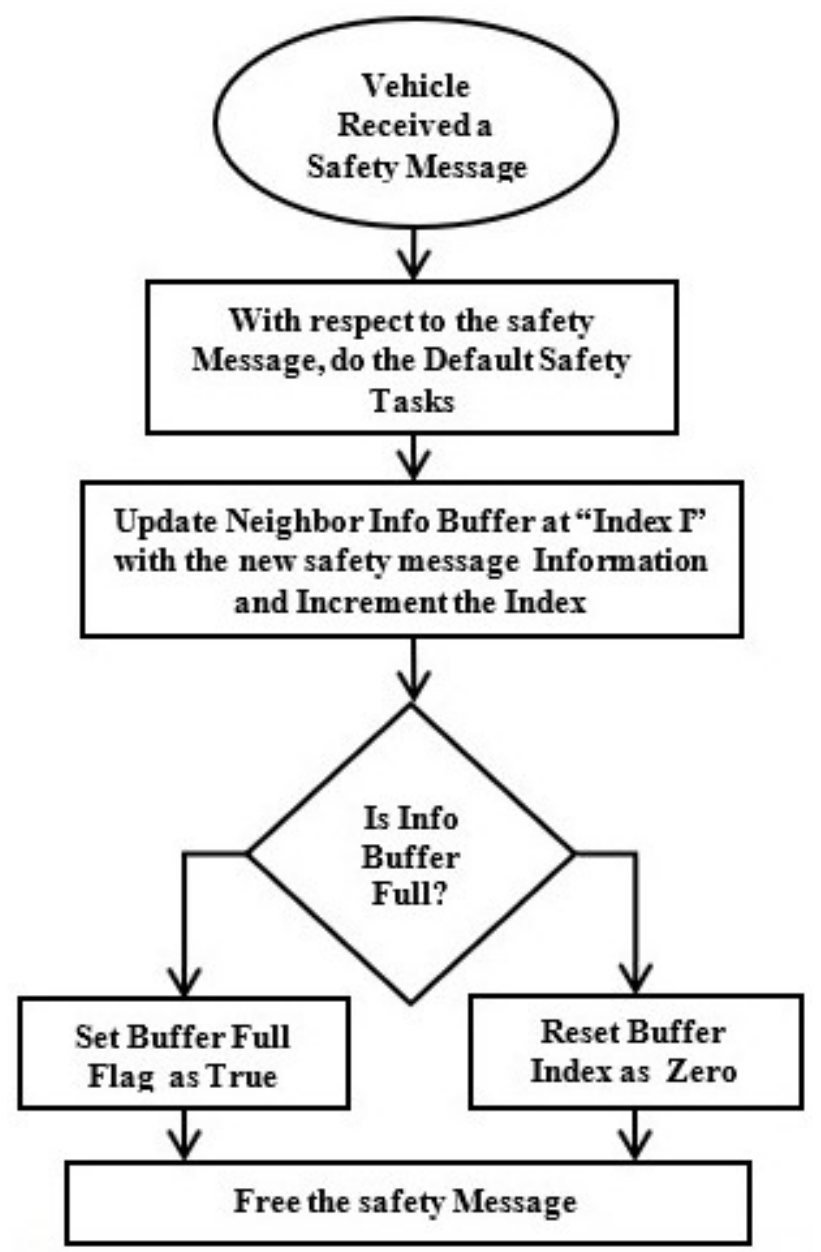

Figure 4 Flowchart for The Receiving End of the Safety Message 


\section{EXPERIMENTAL SETUP}

All evaluation models for the analysis of the proposed algorithms are built using the network simulator (NS2 v-2.35) [35] and VANET Mobisim traffic simulator (VANETMOBISIM) [36]. The performance of the proposed design is implemented in the following types of mobility scenarios:

1: The first scenario is called the Manhattan grid topology, which defines the mobility of vehicles in an urban city/smart city as shown in Figure 5. The set parameter of this scenario is shown in Table 4, which demonstrates the information related to the vehicles that are moving in the urban city. VANETMOBISIM provides a realistic movement that helps in studying the movement of cars on the road. Deployed (20-100) vehicles are moving at different speeds $(15-30 \mathrm{~m} / \mathrm{s})$ during this scenario $(2000 \mathrm{~m} \times 2000 \mathrm{~m})$.

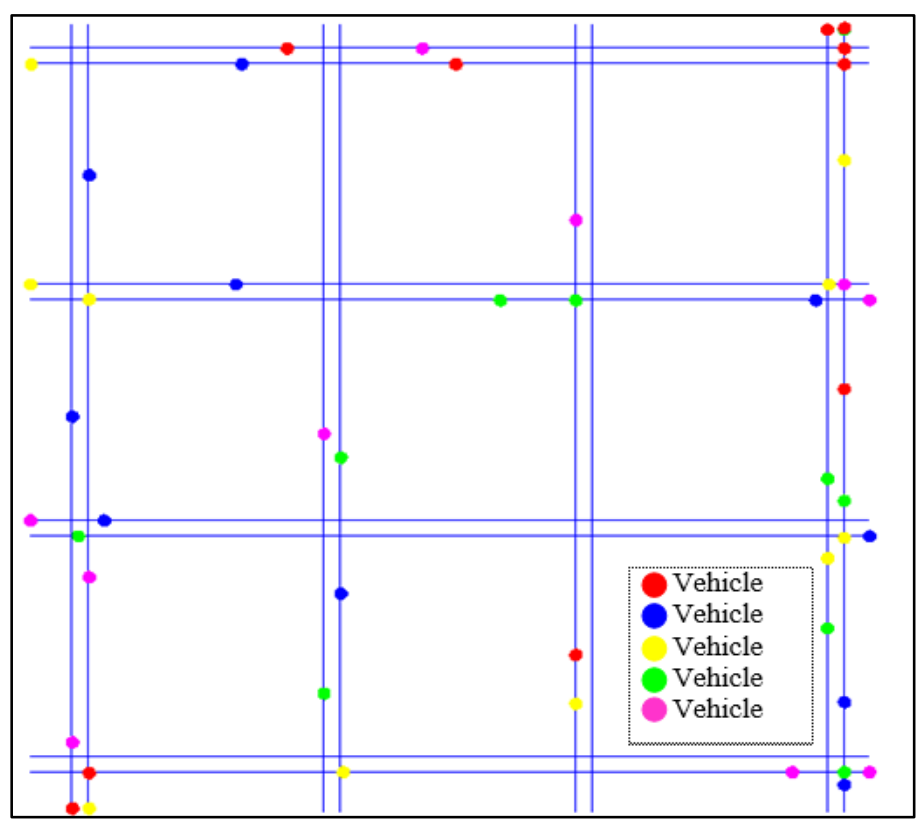

Figure 5 Urban City VANET Simulated Scenario

2: The second scenario is the Freeway topology, which is defined as the mobility of the vehicles at the highway as shown in figure 6. In VANETMOBISIM, creating methods using the needed parameters is easy. Table 4 shows the test methods created for the proposed algorithms to increase road safety during the highway scenario (2000 m long with four lines with (20-100) vehicles moving in different velocities (15-30 m/s). 


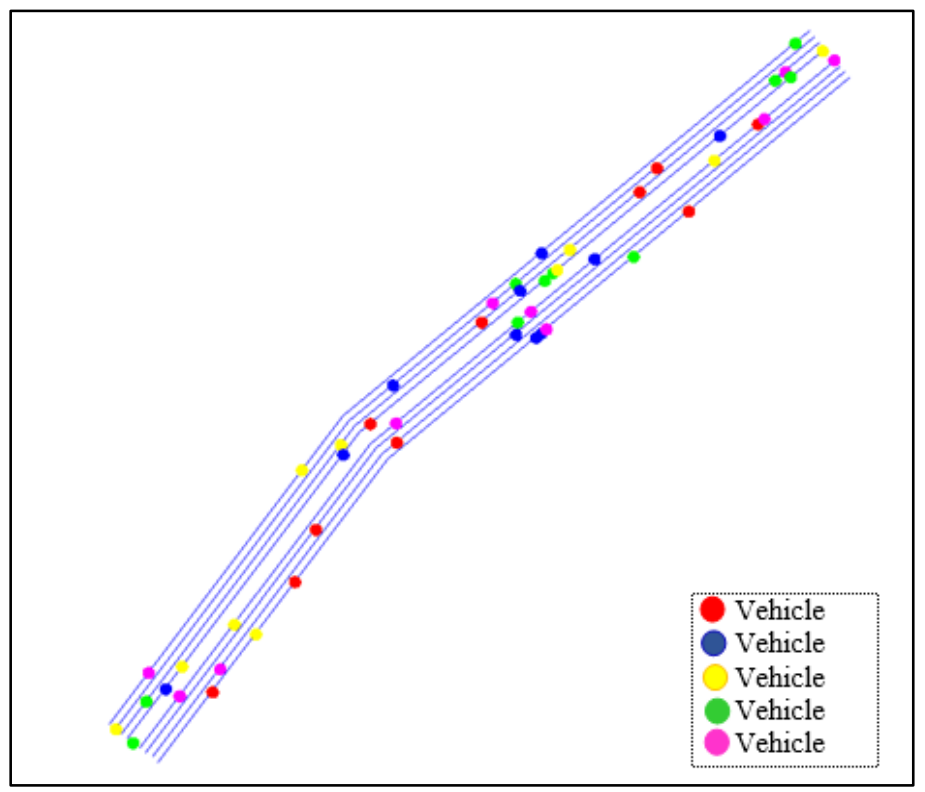

Figure 6 Highway VANET Simulated Scenario

Table 5 contains the significant NS2(v-2.35) parameters used during testing of the proposed algorithms under the NS2(v-2.35), table 5 also contains some important DSRC/802.11p physical and MAC layer parameter settings under the NS2(v-2.35) simulator.

Safety Messages Sent (Nos): The total number of sent safety messages, which are used as a metric to measure the performance of message generation as shown in Equation (5).

$$
\text { Nos }=\sum S_{i}(t)
$$

Where:

$S_{i}(t)=$ total number of generated safety messages at unit time.

Safety Messages Received (Nor): The total number of received safety messages, which are used as a metric to measure the performance of successful message delivery, as shown in Equation (6).

$$
\text { Nor }=\sum S_{i}(t)-\sum D_{i}(t)
$$

Where:

$D_{i}(t)=$ total dropped safety messages at unit time.

$S_{i}(t)=$ total number of generated safety messages at unit time.

Network Throughput (kbps): The ratio of "Size of Total Safety Messages Received" to the duration of the communication, as shown in Equation (7).

Where:

$$
T_{i}(t)=\frac{R_{i}(t)}{t_{i}}
$$

$R_{i}(t)$ : Size of the total number of safety messages received

$t_{i}$ : Total communication time. 
Dropped Message: The difference between the total safety messages sent by the source node and the received message by the destination. The expression of a dropped message is shown in Equation (8) as follows:

$$
\text { Dropped Messages } D=S_{i}(t)-R_{i}(t)
$$

Network Connectivity (\%): The ratio of "Total Safety Messages Generated" to the "Total Safety Messages Successfully Received” at its one-hop neighbors, as shown in Equation (9).

$$
\text { Network connectivity }(\%)=\frac{R_{i}(t)}{S_{i}(t)} \times 100 \% \text {. }
$$

\begin{tabular}{|c|c|c|c|c|c|}
\hline $\begin{array}{l}\text { Mobisim Paramete } \\
\text { VANET }\end{array}$ & for Urban City & $\begin{array}{l}\text { Mobisim Parameters } \\
\text { VANET }\end{array}$ & r Highway & Other parameters of $\mathrm{NS2}(\mathrm{v}-$ & 35) Simulation \\
\hline Parameter & Value & Parameter & Value & Parameter & Value \\
\hline Models & Manhattan & Models & Free Way & $\begin{array}{l}\text { The Periodic Safety Message } \\
\text { Application Simulation } \\
\text { Agent }\end{array}$ & $\mathrm{PBC}$ \\
\hline Range Algorithms & $\begin{array}{l}\text { Null Power } \\
\text { Algorithm }\end{array}$ & Range Algorithms & $\begin{array}{l}\text { Null Power } \\
\text { Algorithm }\end{array}$ & Modulation Schemes & $\begin{array}{l}\text { QAM16 And } 1 / 2 \text { Rate: } 24 \\
\text { Mbps }\end{array}$ \\
\hline Dir12lanespace & 20 & Dir12space & 20 & Broadcast Variance & 0.05 \\
\hline Line num. & 2 & Dir1,2lanespace & 10 & Safety Message Size & 100 \\
\hline Max Acceleration & 2 & Max Acceleration & 2 & Number of Vehicles & $20,40,60,80,100$ \\
\hline $\begin{array}{l}\text { Positive } \\
\text { Acceleration Ratio }\end{array}$ & 2.0 & $\begin{array}{l}\text { Positive Acceleration } \\
\text { Ratio }\end{array}$ & 2.0 & Interface Queue & Drop Tail / Pri-queue \\
\hline $\begin{array}{l}\text { Max Simulation } \\
\text { Time }\end{array}$ & 300 & $\begin{array}{l}\text { Max Simulation } \\
\text { Time }\end{array}$ & 300 & Queue Length & 20 \\
\hline Max Pause Time & 10 & Max Pause Time & 10 & Mac & $\begin{array}{l}\text { Default } 802.11 \mathrm{p}, 802.11 \mathrm{p} \\
\text { with BLTPC, } 802.11 \mathrm{p} \text { with } \\
\text { DTPC }\end{array}$ \\
\hline
\end{tabular}

Table 4 VANETMOBISIM Parameters in Urban City and Highway Scenarios and Some NS2(v-2.35) Parameters

\begin{tabular}{|c|c|c|c|}
\hline \multicolumn{2}{|c|}{ Important DSRC/802.11p MAC Parameter Settings } & \multicolumn{2}{|c|}{ Important DSRC/802.11p PHY Parameter Settings } \\
\hline Parameter & Value & Parameter & Value \\
\hline Cw. min_ & 15 & PHY Carrier sense Thresh & $3.162 \mathrm{e}-12(-85 \mathrm{dBm})$ \\
\hline Cw. max & 1023 & PHY Pt & $\begin{array}{l}\text { Calculated Based on The Necessary Tx } \\
\text { Distance }\end{array}$ \\
\hline Slot time_ & 0.000013 & PHY freq. & $5.9 \mathrm{e}+9$ \\
\hline SIFS_ & 0.000032 & PHY noise floor & 1.26e-13 (-99 dBm for 10mhz Bandwidth) \\
\hline Short retry limit_ & 7 & PHY L & 1.0 (Default Radio Circuit Gain/Loss) \\
\hline Long retry limit_ & 4 & PHY Power Monitor Thresh & $\begin{array}{l}\text { 6.310e-14 ( } 102 \mathrm{dbm} \text { Power Monitor } \\
\text { Sensitivity) }\end{array}$ \\
\hline Header duration_ & 0.000040 & PHY HeaderDuration & 0.000040 \\
\hline Symbol duration_ & 0.000008 & PHY BasicModulationScheme_ & $0,1,2,3$ \\
\hline Basic modulation scheme_ & $0,1,2,3$ & PHY Preamble Capture Switch & 1 \\
\hline Use_802_11a_Flag_ & True & PHY Data Capture Switch & 0 \\
\hline RTS Threshold_ & 2346 & PHY SINR Preamble Capture & $2.5118(4 \mathrm{Db})$ \\
\hline MAC_DBG & 0 & PHY SINR Data Capture_ & $100.0(10 \mathrm{Db})$ \\
\hline Logbackoff & 1 & PHY trace dist._ & 500 \\
\hline
\end{tabular}

Table 5 Important DSRC/802.11p Parameters for MAC/PHY Settings 


\section{PERFORMANCE EVALUATION}

\section{A. Number of Safety message received Analysis}

Figure 7.shows the total numbers of received safety messages in Highway and Urban City VANET network scenarios. During this test, the default transmission range is initiated to be $250 \mathrm{~m}$, modulation scheme QAM16 with $1 / 2$ coding rate is used, speed is $20 \mathrm{~m} / \mathrm{s}$, and safety message interval is 0.1 with safety message size of 100 bytes. Six plots are available: three plots for the highway and three plots for the urban city. Each plot corresponds to different transmission power control algorithms (802.11p MAC, BL-TPC MAC, and DTPC MAC). The plots show that the BLTPC and DTPC algorithms provide better performance than the default MAC algorithm for both scenarios because the designed algorithms (BL-TPC and DTPC) handle the required power dynamically rather than $802.11 \mathrm{p} \mathrm{MAC}$, which deals with transmission power steadily. Moreover, these plots reveal that the DTPC $802.11 \mathrm{p}$ MAC increases the number of received messages to 94\% compared with the traditional 802.11p MAC. Meanwhile, BLTPC 802.11p MAC increases the number of received messages to $50 \%$ compared with the traditional MAC $802.11 \mathrm{p}$. This finding is due to DTPC, which is more flexible than BLTPC considering power management.

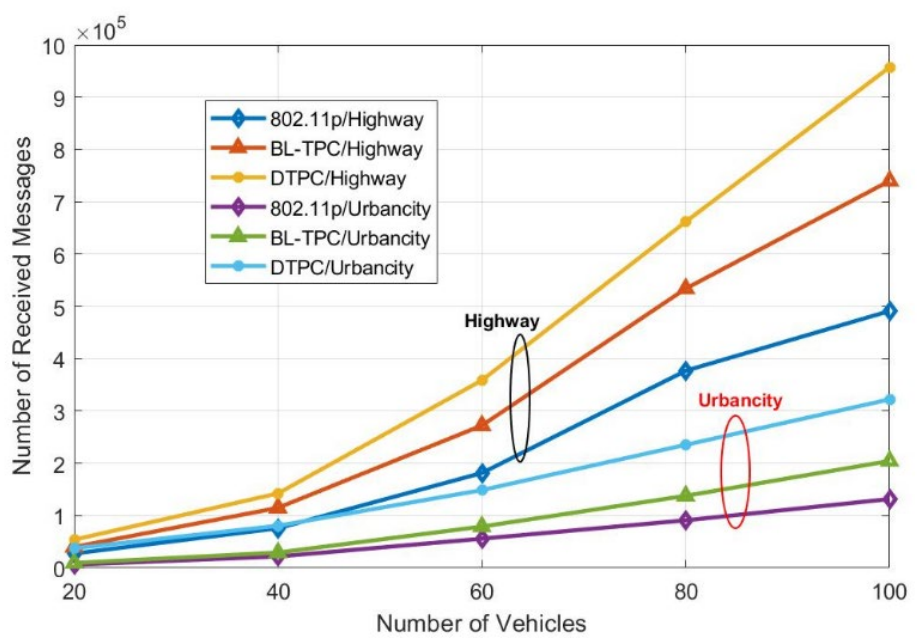

Figure 7 Total number of Received safety Messages vs. Number of vehicles on Highway and Urban city Scenarios

\section{B. Network Connectivity Analysis}

Figure 8 shows the one-hop network connectivity versus the number of vehicles in highway and urban city scenarios. During this test, the default transmission range is initiated to be $250 \mathrm{~m}$, safety message size is 100 bytes, modulation scheme QAM16 with $1 / 2$ coding rate is used, speed is $20 \mathrm{~m} / \mathrm{s}$, and safety message interval is 0.1. Six plots are available: three plots for the highway and three plots for the urban city/smart city. Each plot corresponds to different transmission power control algorithms (802.11p MAC, BL-TPC MAC, and DTPC MAC). The figure depicts that with an increasing number of vehicles, the BL-TPC and DTPC performance is better than the performance of $802.11 \mathrm{p}$ MAC because it reached $99.7 \%$ and $99.5 \%$ connectivity at 100 vehicle density for DTPC and BLTPC, respectively, in both scenarios. The BL-TPC and DTPC power control algorithms can efficiently deal with rapid network topology changes by dynamically varying transmission power according to network topology. Moreover, the figure shows that the change in the number of nodes slightly affects network connectivity using the designed power control algorithms. 


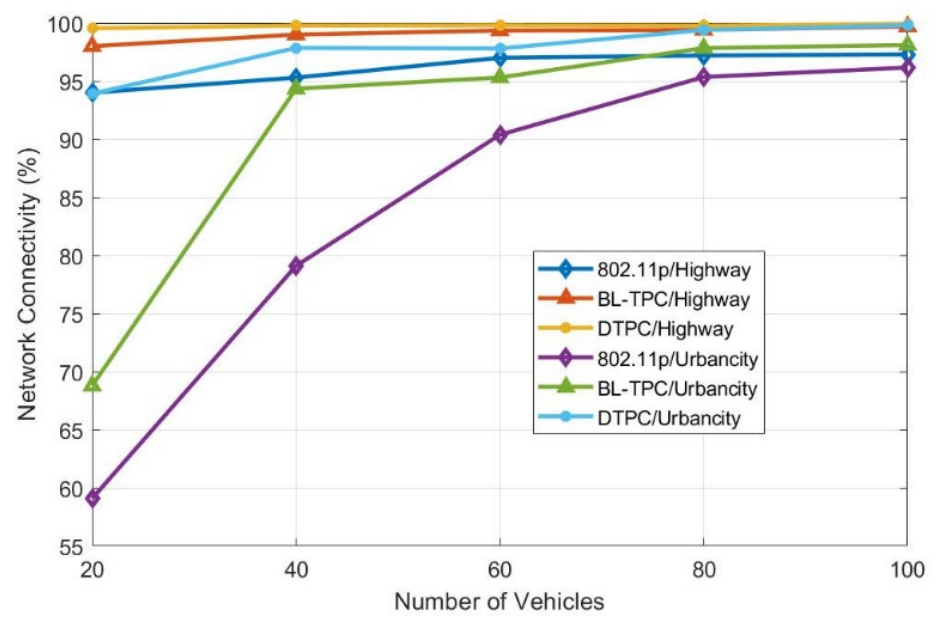

Figure 8 Network Connectivity at Highway and Urban City Scenarios

Figure 9 shows the one-hop network connectivity and the number of received messages versus vehicle speed in an urban city scenario. During this test, the transmission range initiates to $250 \mathrm{~m}$, the number of vehicles is maintained at 60 , and the safety message interval is set as 0.1 . Six plots are available: three plots for network connectivity and three plots for a number of the received messages. Each plot corresponds to different transmission power control algorithms (802.11p MAC, BL-TPC MAC, and DTPC MAC). The figure demonstrates that the designed power control algorithms (BL-TPC and DTPC) provide effective adaptation to fast network topology changes. This result leads to fast maintenance in network connectivity than that in traditional 802.11p MAC because the designed algorithms improve the network connectivity to 98\% compared with traditional 802.11p MAC. By contrast, the figure shows that the increase in vehicle speed has a slight effect on network connectivity when using DTPC and BLTPC algorithms. Figure 9 also shows the total numbers of received safety messages in the VANET network versus vehicle speed. The figure demonstrates that the performance of DTPC and BL-TPC outperforms that of the traditional power control algorithm 802.11p MAC with an increase in vehicle speed. By contrast, the figure shows that the number of received safety message increases when using BL-TPC and DTPC with an increase in vehicle speed. Meanwhile, the number of received safety message decreases when the vehicle speed is increased without any algorithm. The BL-TPC and DTPC can change the transmission power dynamically according to the vehicle density, while the traditional 802.11p MAC uses fixed power and cannot deal with the nontrivial task of maintaining connectivity due to rapid topology changes.

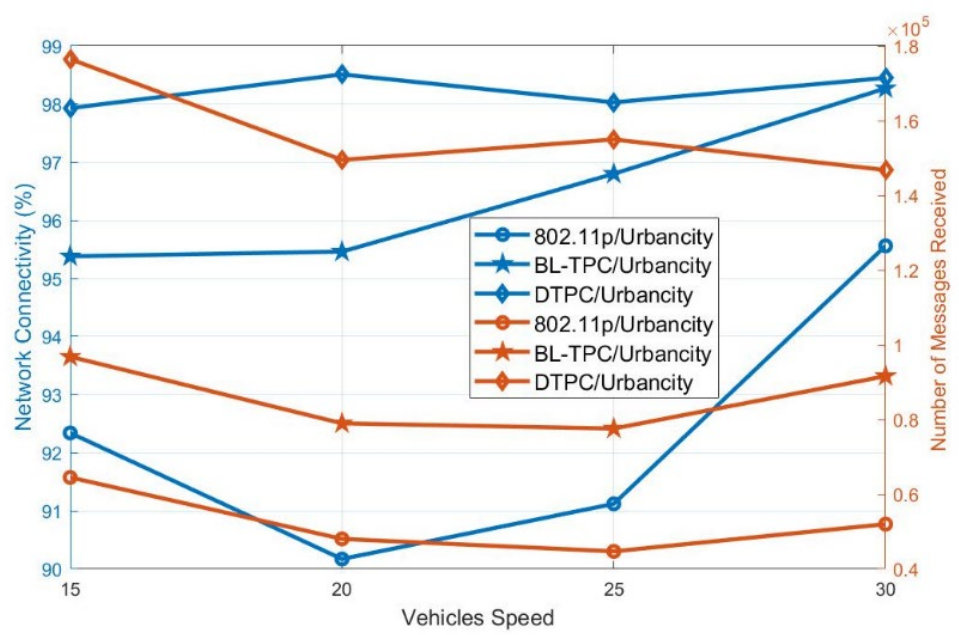

Figure 9 Total Number of Received Safety Messages and Network Connectivity vs. Different Vehicle Speed 


\section{Throughput Analysis}

Figure 10 shows the average network throughput versus the number of nodes in two different VANET scenarios (Highway and Urban City). During this test, the default transmission range is $250 \mathrm{~m}$, modulation scheme QAM16 with $1 / 2$ coding rate is used, safety message size is 100 bytes, speed is $20 \mathrm{~m} / \mathrm{s}$, and safety message interval is 0.1 . Six plots are available: three plots for highway and three plots for the urban city/smart city, in which each plot corresponds to different power control algorithms (802.11p MAC, BL-TPC MAC, and DTPC MAC). The figure depicts that the average network throughput using the designed power control algorithm outperforms that using the traditional algorithm (fixed power algorithm). This finding is because BL-TPC and DTPC can maintain network connectivity flexibly by changing transmission power dynamically according to network topology. Using dynamic transmission power improves network connectivity, which leads to a decrease in the number of lost messages and increases network throughput.

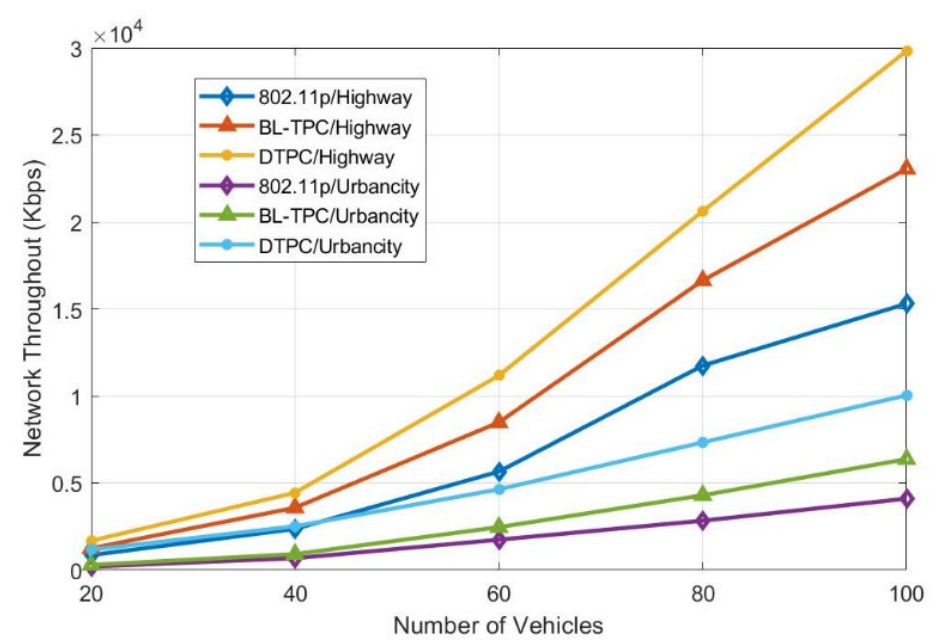

Figure 10 Network Throughput in Highway and Urban City Scenarios

\section{Number of Dropped Messages Analysis}

Figure 11 shows the number of dropped messages versus the number of vehicles in two different VANET scenarios (highway and urban city). During this test, the default transmission range is $250 \mathrm{~m}$, safety message size is 100 bytes, modulation scheme QAM16 with $1 / 2$ coding rate is used, speed is $20 \mathrm{~m} / \mathrm{s}$, and safety message interval is 0.1 . Six plots are available: three plots for highway and three plots for the urban city. Each plot corresponds to different power control algorithms (802.11p MAC, BL-TPC MAC, and DTPC MAC). Figure 11 shows that the number of lost messages increases with vehicle number due to node contention. Moreover, the figure demonstrates that the number of lost messages when using BL-TPC and DTPC is less than that using the traditional $802.11 \mathrm{p} \mathrm{MAC}$ algorithm. This finding is due to the dynamic changes in power using BL-TPC and DTPC according to node density. By contrast, the power is kept fixed using $802.11 \mathrm{p}$ MAC rather than the node density. 


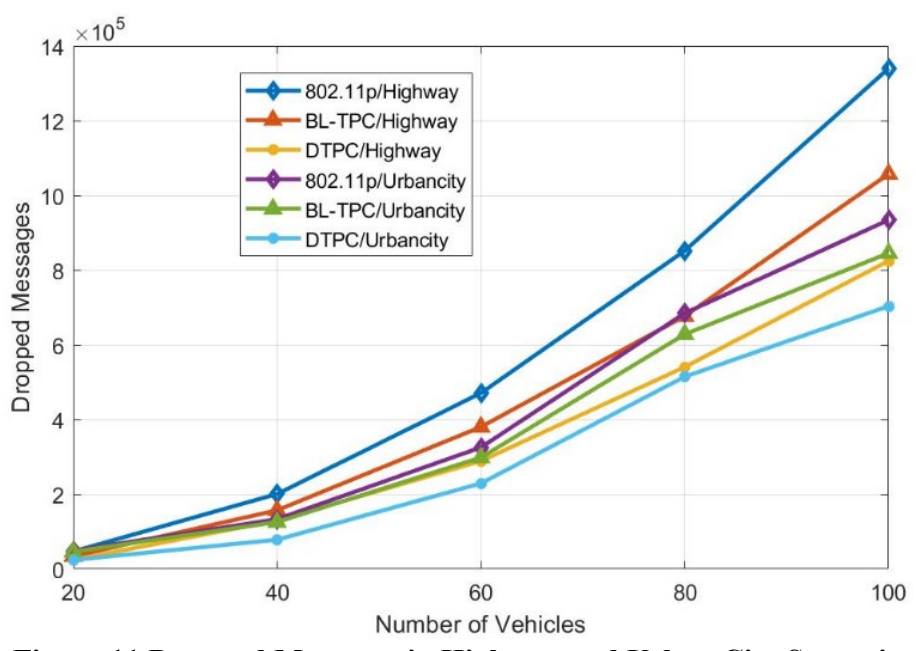

Figure 11 Dropped Messages in Highway and Urban City Scenarios

Figure 12 shows the number of dropped messages versus vehicle speed in an urban city scenario. During this test, the transmission range is $250 \mathrm{~m}$, the number of vehicles is maintained at 60 , and the safety message interval is set to 0.1 . Three plots are available, and each plot corresponds to different power control algorithms (802.11p MAC, BL-TPC MAC, and DTPC MAC). The figure demonstrates that the designed power control algorithms (BL-TPC and DTPC) provide better performance than the traditional one 802.11p MAC. The BLTPC and DTPC algorithms can dynamically adapt transmission power according to network topology and demonstrate fast and continuous changes due to high vehicle speed. By contrast, the figure shows that increasing vehicle speed has a slight effect on network throughput when using DTPC and BLTPC algorithms. Furthermore, the figure demonstrates that the number of lost messages using BL-TPC and DTPC is less than that using the traditional 802.11p MAC power algorithm. Moreover, the BL-TPC and DTPC algorithms decrease the loss of messages by $9 \%$ and $40 \%$, respectively, because both designed algorithms are based on dynamic changes in transmission power according to node density and network topology.

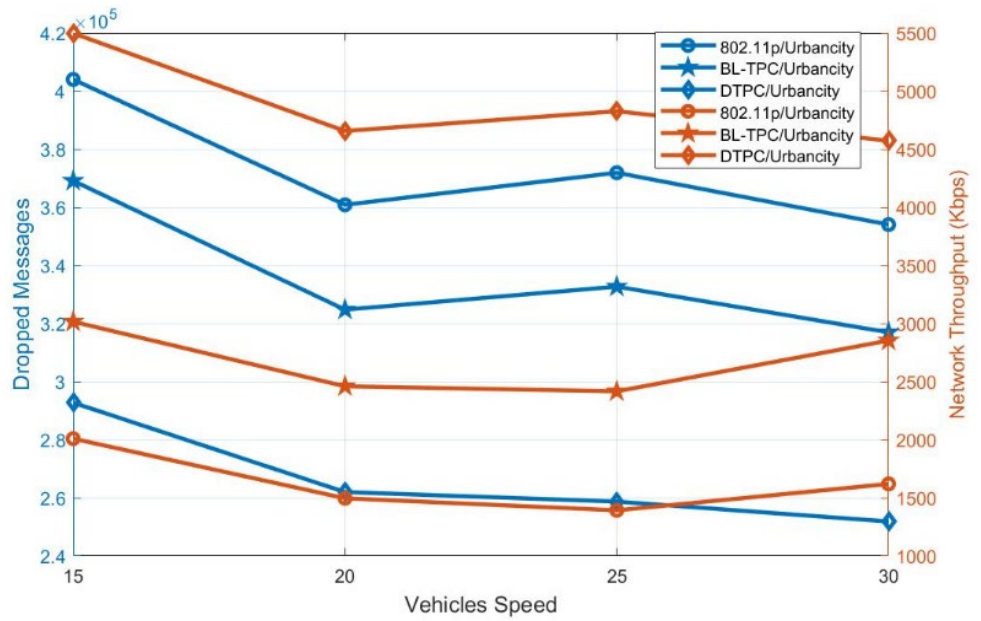

Figure 12 Dropped Messages and Network Throughput vs Different Vehicle Speed

\section{COMPARISON}

As previously mentioned in this paper, the network connectivity is the most important factor that affects the performance of the VANET. Therefore, achieving high network connectivity leads to improved network 
throughput and packet delivery ratio and decreased number of lost packets. Network connectivity is affected by network density and node speed. This section presents a simple comparison between the developed algorithms and some other power control algorithms demonstrated in related work. Table 6 shows that the developed algorithms adapt the power to maintain network connectivity, and the connectivity is nearly fixed despite the increase in vehicle density or speed. This finding is because the design of the developed algorithms is based on an estimation network environment (density and speed) at the application layer and then adapts transmission power at PHY/MAC layers. Meanwhile, the network connectivity using other algorithms strongly decreases at increasing vehicle speed because its design is based on routing protocols. Therefore, speed and density are crucial parameters that affect network connectivity.

Table 6 Comparison between the previous algorithms and the designed algorithms

\begin{tabular}{|c|c|c|c|c|c|c|c|c|}
\hline Reference & $\begin{array}{l}\text { Algorithm } \\
\text { Name }\end{array}$ & $\begin{array}{l}\text { Modification } \\
\text { Layers }\end{array}$ & $\begin{array}{l}\text { Propagatio } \\
\text { n type }\end{array}$ & Tested area & $\begin{array}{l}\text { Modified } \\
\text { protocol }\end{array}$ & $\begin{array}{l}\text { Network } \\
\text { connectivity } \\
\text { at node } \\
\text { density } 30 \text { - } \\
\text { 50) vehicles }\end{array}$ & $\begin{array}{l}\text { Network } \\
\text { Performance } \\
\text { when increase } \\
\text { node density } \\
\text { more than } 50 \\
\text { vehicle }\end{array}$ & $\begin{array}{l}\text { Network } \\
\text { Performance } \\
\text { when increase } \\
\text { node speed }\end{array}$ \\
\hline [13] & $\begin{array}{l}\text { ATP- } \\
\text { ADOV }\end{array}$ & Network & Broadcast & $\begin{array}{l}\text { Highways } \\
\text { and Urban } \\
\text { City }\end{array}$ & AODV & $95 \%$ & Decrease & $\begin{array}{l}\text { Strongly } \\
\text { Decrease }\end{array}$ \\
\hline [17] & CLPC & $\begin{array}{l}\mathrm{PHY} / \mathrm{MAC} \text { and } \\
\text { Network. }\end{array}$ & $\begin{array}{l}\text { Two Ray } \\
\text { Model }\end{array}$ & Square area & IEEE 802.11 & $93-95 \%$ & Decrease & $\begin{array}{l}\text { Strongly } \\
\text { Decrease }\end{array}$ \\
\hline $\begin{array}{l}\text { Our } \\
\text { Algorithms }\end{array}$ & $\begin{array}{l}\text { DTPC } \\
\& \\
\text { BLTPC }\end{array}$ & $\begin{array}{l}\mathrm{PHY} / \mathrm{MAC} \\
\text { and } \\
\text { Application }\end{array}$ & $\begin{array}{l}\text { Nakagami } \\
\text { propagation }\end{array}$ & $\begin{array}{l}\text { Highways } \\
\text { and Urban } \\
\text { City }\end{array}$ & IEEE $802.11 \mathrm{p}$ & $98-99 \%$ & Nearly Fixed & Nearly Fixed \\
\hline
\end{tabular}

\section{CONCLUSION}

VANETS are developed as a part of the ITS. This type of network is a standalone network that works without the internet. Each node can send and receive messages with neighbors that are located within its transmission range. In VANET, the density of the vehicles frequently and rapidly changes due to high node mobility, which leads to changes in vehicle distribution (change network topology) and the connectivity graph. Therefore, transmission power is crucial in maintaining network connectivity. New power control algorithms were designed, modeled, and evaluated in this paper. The design of developed algorithms is based on a crosslayer design between application, mac, and physical layers. The design algorithms were named as BLTPC and DTPC. Both algorithms can change transmission power dynamically according to network node density to maintain connectivity between vehicles on the road. The obtained results show that the designed power control algorithms improve network connectivity between the vehicle in dense and sparse regions. Meanwhile, network throughput and the number of lost messages are improved. Our future works will address the issues in the design of dynamic, optimum transmission power control algorithms by reducing possible message overheads. If one algorithm can set an optimum transmission power level at each node in the VANET without increasing message overheads, then this algorithm will improve the network connectivity with respect to minimize the overload messages and dropped message under VANET and show the ways to improve the performance little further. Thus, the performance of normal one-hop safety messages and multi-hop communication will be improved . 


\section{REFERENCES}

[1] W. Wu, Z. Yang, and K. Li, “16 16.1,” pp. 299-317.

[2] E. C. Eze, S. J. Zhang, E. J. Liu, and J. C. Eze, “Advances in vehicular ad-hoc networks (VANETs): Challenges and road-map for future development," Int. J. Autom. Comput., vol. 13, no. 1, pp. 1-18, 2016.

[3] C. M. Raut and S. R. Devane, "Intelligent transportation system for smartcity using VANET," Proc. 2017 IEEE Int. Conf. Commun. Signal Process. ICCSP 2017, vol. 2018-Janua, pp. 1602-1605, 2018.

[4] B. Chen and H. H. Cheng, "A review of the applications of agent technology in traffic and transportation systems," IEEE Trans. Intell. Transp. Syst., vol. 11, no. 2, pp. 485-497, 2010.

[5] F. Camacho, C. Cárdenas, and D. Muñoz, "Emerging technologies and research challenges for intelligent transportation systems: 5G, HetNets, and SDN," Int. J. Interact. Des. Manuf., vol. 12, no. 1, pp. 327-335, 2018.

[6] H. Hakim, H. Boujemaa, and W. Ajib, "Performance comparison between adaptive and fixed transmit power in underlay cognitive radio networks," IEEE Trans. Commun., vol. 61, no. 12, pp. 4836-4846, 2013.

[7] S. Lin et al., "ATPC: Adaptive transmission power control for wireless sensor networks," ACM Trans. Sens. Networks, vol. 12, no. 1, pp. 223-236, 2016.

[8] S. Sulistyo and S. Alam, "Distributed channel and power level selection in VANET Based on SINR using game model," Int. J. Commun. Networks Inf. Secur., vol. 9, no. 3, pp. 432-438, 2017.

[9] J. A. Sanguesa et al., "Vehicle density and roadmap topology issues when characterizing vehicular communications," Proc. - 2015 IEEE 14th Int. Symp. Netw. Comput. Appl. NCA 2015, pp. 200-203, 2016.

[10] X. Hou, Y. Li, D. Jin, D. O. Wu, and S. Chen, "Modeling the impact of mobility on the connectivity of vehicular networks in large-scale urban environments," IEEE Trans. Veh. Technol., vol. 65, no. 4, pp. 2753-2758, 2016.

[11] A. A. Almohammedi, N. K. Noordin, and S. Saeed, "Evaluating the impact of transmission range on the performance of VANET," Int. J. Electr. Comput. Eng., vol. 6, no. 2, pp. 800-809, 2016.

[12] O. I. Khalaf, G. M. Abdulsahib, and M. Sadik, “A modified algorithm for improving lifetime in WSN,” J. Eng. Appl. Sci., vol. 13, no. 21, pp. 9277-9282, 2018.

[13] M. Izharul, S. P. Singh, and M. N. Doja, “Adaptive-Transmission-Power Ad Hoc On-Demand Distance Vector Routing Protocol for Mobile Ad hoc Network," Int. J. Comput. Appl., vol. 138, no. 2, pp. 1-10, 2016.

[14] M. Abdelgadir, R. A. Saeed, and A. Babiker, "Cross Layer Design Approach for Efficient Data Delivery Based on IEEE 802.11P in Vehicular Ad-Hoc Networks (VANETS) for City Scenarios,” Int. J. AdHoc Netw. Syst., vol. 8, no. 4, pp. 01-12, 2018.

[15] A. Sarfaraz Ahmed, T. Senthil Kumaran, S. Syed Abdul Syed, and S. Subburam, "Cross-layer design approach for power control in mobile ad hoc networks," Egypt. Informatics J., vol. 16, no. 1, pp. 1-7, 2015.

[16] K. Kunavut, “A Novel Adaptive Topology Control in Mobile Ad Hoc Networks based on Connectivity Index,” pp. 87-91, 2015.

[17] D. B. Rawat, D. C. Popescu, G. Yan, and S. Olariu, "Enhancing VANET performance by joint adaptation of transmission power and contention window size," IEEE Trans. Parallel Distrib. Syst., vol. 22, no. 9, pp. 1528-1535, 2011.

[18] H. Chang, Y. E. Song, H. Kim, and H. Jung, "Distributed transmission power control for communication congestion control and awareness enhancement in VANETs," PLoS One, vol. 13, no. 9, pp. 1-25, 2018.

[19] X. W. Wu, W. Yan, S. M. Song, and H. Bin Wang, "A transmission range adaptive broadcast algorithm for vehicular ad hoc networks," NSWCTC 2010 - 2nd Int. Conf. Networks Secur. Wirel. Commun. Trust. Comput., vol. 1, pp. 28-32, 2010.

[20] P. J. Wan, C. W. Yi, and L. Wang, "Asymptotic critical transmission radius for א-connectivity in wireless ad hoc networks," IEEE Trans. Inf. Theory, vol. 56, no. 6, pp. 2867-2874, 2010.

[21] S. A. Ali Shah et al., "Coverage Differentiation Based Adaptive Tx-Power for Congestion and Awareness Control in VANETs," Mob. Networks Appl., vol. 23, no. 5, pp. 1194-1205, 2018.

[22] Y. Zhuang, J. Pan, Y. Luo, and L. Cai, "Time and location-critical emergency message dissemination for Vehicular Ad-hoc Networks," IEEE J. Sel. Areas Commun., vol. 29, no. 1, pp. 187-196, 2011.

[23] B. J. B. Kenney, "Dedicated Short-Range Communications ( DSRC ) Standards in the United States," vol. 99, no. 7, 2011.

[24] X. Ma and X. Chen, "Delay and broadcast reception rates of highway safety applications in vehicular ad hoc networks," 2007 Mob. Netw. Veh. Environ. MOVE, pp. 85-90, 2007.

[25] X. Wu et al., "Vehicular communications using DSRC: Challenges, enhancements, and evolution," IEEE J. Sel. Areas Commun., vol. 31, no. 9, pp. 399-408, 2013.

[26] D. Jiang, V. Taliwal, A. Meier, W. Holfelder, and R. Herrtwich, "Design of 5.9 GHz DSRC-based vehicular safety communication," IEEE Wirel. Commun., vol. 13, no. 5, pp. 36-43, 2006.

[27] D. Jiang and L. Delgrossi, "IEEE 802.11p: Towards an international standard for wireless access in vehicular environments," IEEE Veh. Technol. Conf., pp. 2036-2040, 2008.

[28] C. Cooper, D. Franklin, M. Ros, F. Safaei, and M. Abolhasan, "A Comparative Survey of VANET Clustering Techniques," IEEE Commun. Surv. Tutorials, vol. 19, no. 1, pp. 657-681, 2017.

[29] B. E. Bilgin and V. C. Gungor, "Performance comparison of ieee 802.11p and IEEE 802.11b for vehicle-to-vehicle communications in highway, rural, and urban areas," Int. J. Veh. Technol., vol. 2013, 2013.

[30] Y. L. Morgan, "Notes on DSRC \& WAVE standards suite: Its architecture, design, and characteristics," IEEE Commun. Surv. Tutorials, vol. 12, no. 4, pp. 504-518, 2010.

[31] G. Karagiannis et al., "Vehicular networking: A survey and tutorial on requirements, architectures, challenges, standards and solutions," IEEE Commun. Surv. Tutorials, vol. 13, no. 4, pp. 584-616, 2011.

[32] Y. Wang, J. Hu, Y. Zhang, and C. Xu, "Reliability evaluation of IEEE 802.11 p-based vehicle-to-vehicle communication in an urban expressway,” Tsinghua Sci. Technol., vol. 20, no. 4, pp. 417-428, 2015.

[33] S. A. M. Ahmed, S. H. S. Ariffin, and N. Fisal, "Overview of wireless access in vehicular environment (wave) protocols and standards," Indian J. Sci. Technol., vol. 6, no. 7, pp. 4994-5001, 2013.

[34] F. J. Martinez, C. K. Toh, J. C. Cano, C. T. Calafate, and P. Manzoni, "Realistic radio propagation models (RPMs) for VANET simulations," IEEE Wirel. Commun. Netw. Conf. WCNC, 2009. 
[35] Kevin Fall, "The ns Manual-The VINT Project," no. 3, 2011.

[36] U. Guide, "User Guide TradeView User Guide," no. January, 2013.

\section{List of Figures}

Figure 1 DSRC spectrum frequencies and IEEE1609.4 protocol operations ....................................................................5

Figure 2. Flowchart for BL-TPC 802.11p MAC Algorithm Operation Concept infrastructure ..................................................9

Figure 3. Flowchart for DTPC 802.11p MAC Algorithm Operation Concepts.......................................................................11

Figure 4 Flowchart for The Receiving End of the Safety Message ...............................................................................

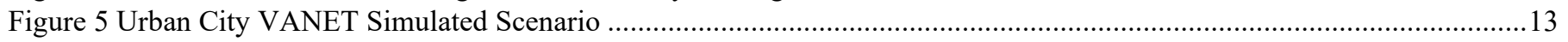

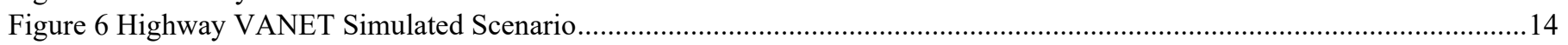

Figure 7 Total number of Received safety Messages vs. Number of vehicles on Highway and Urban city Scenarios..................16

Figure 8 Network Connectivity at Highway and Urban City Scenarios .........................................................................17

Figure 9 Total Number of Received Safety Messages and Network Connectivity vs. Different Vehicle Speed.........................17

Figure 10 Network Throughput in Highway and Urban City Scenarios .........................................................................18

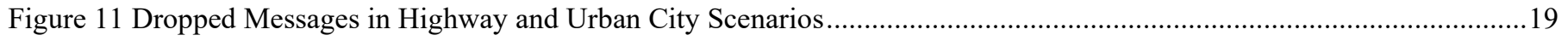

Figure 12 Dropped Messages and Network Throughput vs Different Vehicle Speed .......................................................19

\section{List of Tables}

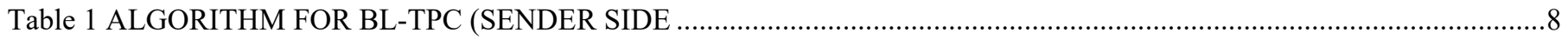

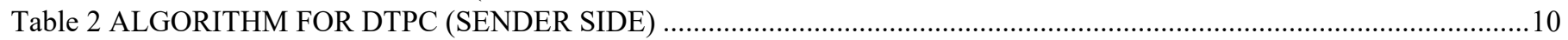

Table 3 ALGORITHM FOR BL-TPC\& DTPC (RECEIVER SIDE) ..............................................................................12

Table 4 VANETMOBISIM Parameters in Urban City and Highway Scenarios and Some NS2(v-2.35) Parameters...................15

Table 5 Important DSRC/802.11p Parameters for MAC/PHY Settings .............................................................................15

Table 6 Comparison between the previous algorithms and the designed algorithms .........................................................20 